\title{
High Prevalence of Deep Vein Thrombosis in Tsunami-Flooded Shelters Established after the Great East-Japan Earthquake
}

\author{
Shinsaku Ueda, ${ }^{1}$ Kazuhiko Hanzawa, ${ }^{2}$ Muneichi Shibata ${ }^{3}$ and Satoshi Suzuki ${ }^{1}$ \\ ${ }^{1}$ Department of Thoracic Surgery, Japanese Red Cross Ishinomaki Hospital, Ishinomaki, Miyagi, Japan \\ ${ }^{2}$ Department of Cardiovascular Surgery, Niigata University Hospital, Niigata, Japan \\ ${ }^{3}$ Department of Cardiology, Miyagi Cardiovascular and Respiratory Center, Kurihara, Miyagi, Japan
}

\begin{abstract}
High prevalence of deep vein thrombosis (DVT) in disaster shelters has been reported in the aftermath of earthquakes in Japan. Calf DVT was examined using sonography in the shelters after the Great East Japan earthquake on March 11, 2011. By the end of July 2011, 701 out of 8,630 evacuees suspected with calf DVT, judged by inspections or medical interviews, were examined in 32 shelters, and 190 evacuees were confirmed to have calf DVT. The prevalence of DVT was $2.20 \%$, which was 200 times higher than the usual incidence in Japan. The DVT prevalence seemed to decrease with time. By the end of May, a significantly higher prevalence of DVT was found in tsunami-flooded shelters (109 of 3,871 evacuees; $2.82 \%$ ) than in non-flooded shelters (53 of 3,155 evacuees; $1.68 \%$ ). After June, its prevalence was still higher $(18 / 541 ; 3.33 \%)$ in tsunami-flooded shelters than in non-flooded shelters $(10 / 1063 ; 0.94 \%)$. The cause of the high prevalence of DVT was supposed to be dehydration due to the delay in supplying drinking water, vomiting, and diarrhea experienced by the evacuees because of a shortage of clean water to wash their hands. Dehydration was especially noticed in women because they restricted themselves of water intake to avoid using unsanitary toilet facilities. Moreover, crowded shelters restricted the mobility of elderly people, which would exacerbate the prevalence of DVT. Those deteriorated and crowded shelters were observed in tsunami-flooded areas. Therefore, long-term shelters should not be set up in flooded areas after tsunami.
\end{abstract}

Keywords: deep vein thrombosis; emergency shelter; Great East Japan Earthquake; health hazard; tsunami Tohoku J. Exp. Med., 2012, 227 (3), 199-202. 다이 2012 Tohoku University Medical Press

After the Great East Japan Earthquake on March 11, 2011 (Shibahara 2011), the casualties of the tsunami were brought to an area near the seashore and downtown of Ishinomaki located at the northeast coast of Honshu Island, Japan (Fig. 1). Following the earthquake, the tsunami flooded about $13 \%$ of the city, where $70 \%$ of the population lived. There were more than 47,000 evacuees from the city. A high incidence of pulmonary thromboembolism (PTE), among the most serious complications of deep vein thrombosis (DVT), has been reported in evacuees in 2004 Niigata Prefecture Chuetsu Earthquake (Inoue 2006; Watanabe et al. 2008). Therefore, the immense damage caused by the tsunami was thought to increase the number of DVT patients, which might result in a high prevalence of PTE. Thus, we examined the evacuees for calf DVT by portable sonography to determine the prevalence of DVT in Ishinomaki.

\section{Methods}

The evacuees in the shelters were asked about swelling of their lower extremities and physical inactivity through the end of July 2011. Then, evacuees suspected with calf DVT were examined using portable sonography (Viamo with $7.5 \mathrm{MHz}$ linear probe; Toshiba Co, Tokyo; MyLabFive with a 4-9 MHz linear probe; Hitachi-Aloka Medical, Ltd., Tokyo; and MicroMaxx with a 5-10 MHz linear probe; SonoSite Japan, Tokyo). The levels of blood D-dimer, a degradation product of fibrin, were measured in evacuees with multiple or free-floating thrombi in their calf veins. Statistical analysis was performed using the chi-square test. The Institutional Review Board of the Japanese Red Cross Ishinomaki Hospital approved this study.

\section{Results and Discussion}

We visited 32 shelters, including 19 shelters directly flooded by the tsunami (flooded shelters), and asked 8,630 evacuees about their symptoms. In flooded shelters, the evacuees stayed upstairs when tsunami attacked the first floor, and most of evacuees remained afterword. We examined 701 candidates for calf DVT and identified DVT

Received April 27, 2012; revision accepted for publication June 11, 2012. doi: 10.1620/tjem.227.199

Correspondence: Shinsaku Ueda, M.D., Japanese Red Cross Ishinomaki Hospital, 71 Nishimichishita-Hebita, Ishinomaki, Miyagi 9868522, Japan.

e-mail: beacon-ueda@onyx.ocn.ne.jp 


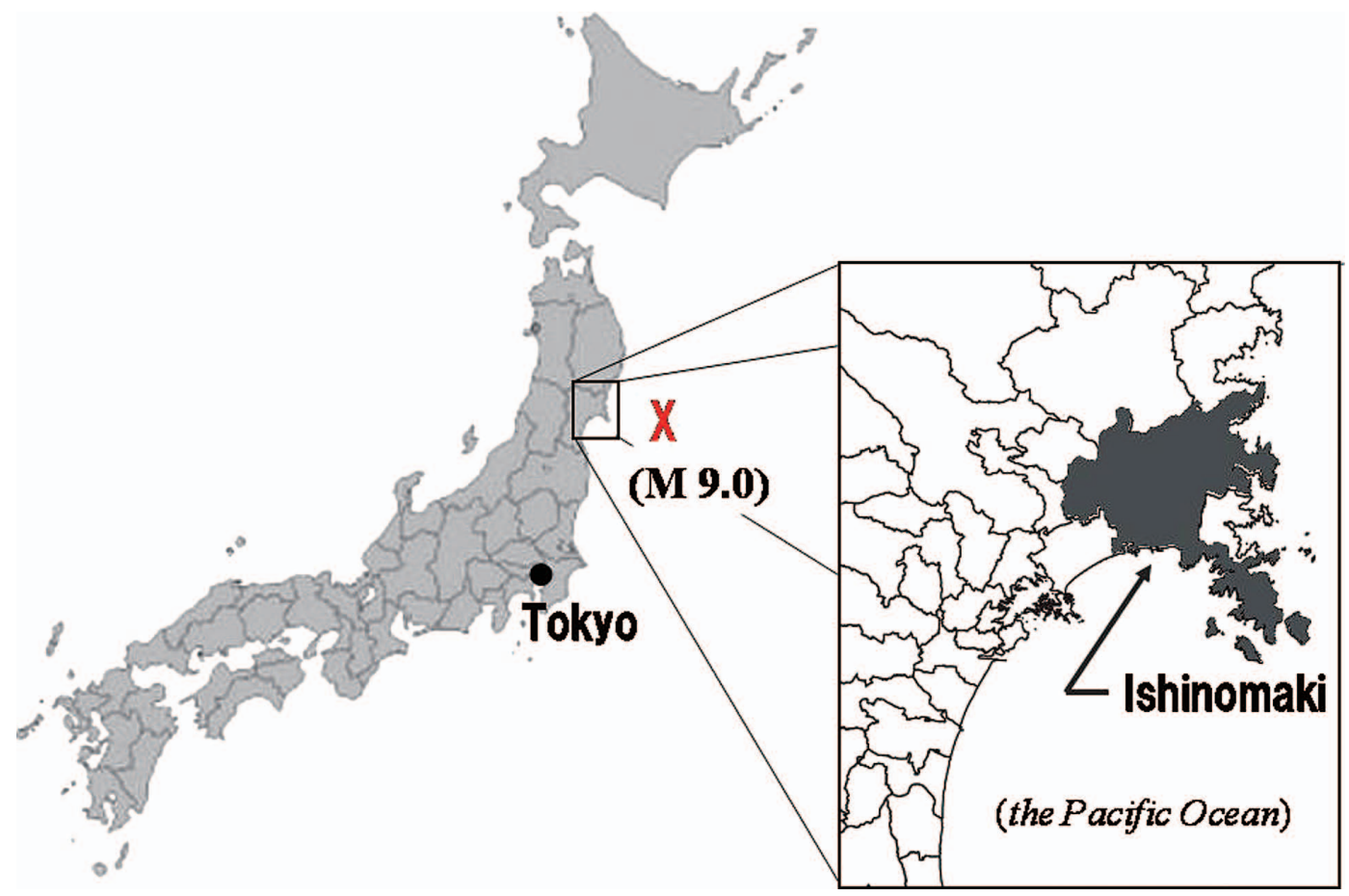

Fig. 1. Ishinomaki City at the pacific coast of northeast Japan.

Shown is the location of Ishinomaki City in Honshu (Main Island) of Japan. The epicenter of the Great East Japan Earthquake in 2011 is indicated at $130 \mathrm{~km}$ off the pacific coast (X).

echoes in 190 evacuees (137 women; 118 older than 70 years), which indicated the prevalence of DVT as $2.20 \%$, approximately 200 times higher than the usual incidence in Japan (Sakuma et al. 2009). It has been reported that 20\% of calf DVTs propagate to proximal veins and can potentially lead to pulmonary embolism (Philbrick and Becker 1988). The usefulness of investigating calf DVT for evaluating the risk of PTE is controversial (Atri et al. 1996), but it was a feasible approach for screening DVT in emergency shelters. In fact, the examination of calf only required the evacuees to roll up their trousers. The accuracy of compression sonography with the addition of Doppler imaging for the diagnosis of calf DVT has been reported (Atri et al. 1996). In some evacuees with multiple or free-floating thrombi, blood D-dimer levels were measured, and 6 of them with levels $>1.0 \mu \mathrm{g} / \mathrm{mL}$ were further examined by whole body computed tomography at the Japanese Red Cross Ishinomaki Hospital. Finally, 2 female evacuees were hospitalized for anti-coagulant therapy for PTE. Others were advised to wear elastic stockings. No evacuee died from DVT. The DVT prevalence in shelters was found to be high soon after the disaster, and seemed to decrease with time (Fig. 2). From March to the end of May, the DVT prevalence was significantly higher in tsunamiflooded shelters (109 of 3,871 evacuees; 2.82\%) than in non-flooded shelters (53 of 3,155 evacuees; $1.68 \%)(P=$ 0.0016). Even after June, the prevalence of DVT was still higher in tsunami-flooded shelters (18 of 541 evacuees; $3.33 \%$ ) as compared to that in non-flooded shelters (10 of
1,063 evacuees; $0.94 \%)(P<0.001)$ (Table 1).

Although the flooding by the tsunami itself had ceased within a few weeks, tsunami destroyed infrastructure and left huge amounts of sludge, which obstructed ground access for repair and transport for several weeks. Very limited supplies of water and food directly caused dehydration, which was exacerbated by vomiting and diarrhea experienced by the evacuees because of a shortage of clean water to wash their hands polluted by mud. Women appeared to minimize their water intake to avoid frequent use of unsanitary toilet facilities in the shelters. High temperature and humidity in June-July might have exacerbated dehydration in places without air conditioning. The elderly might have been immobile for long periods because of difficulty in walking in crowded shelters in tsunami-flooded areas where most of the evacuees lost their houses and remained in the shelters for months. In the tsunami-flooded areas, shelters were very crowded, ie, approximately 1,000 evacuees per shelter. The high prevalence of DVT in tsunami-flooded shelters after June was thought to indicate that the effect that tsunami flooding had on the evacuees would sustain for a long time.

According to the guideline of the Federal Emergency Management Agency (FEMA 2008), the recommended minimum square footage per occupant for a tsunami refuge is 10 square feet per person for a short stay, approximately 24 hours. It would not be considered appropriate for a longer stay. This guideline also states that the American Red Cross recommends a minimum of 20 square feet per person 


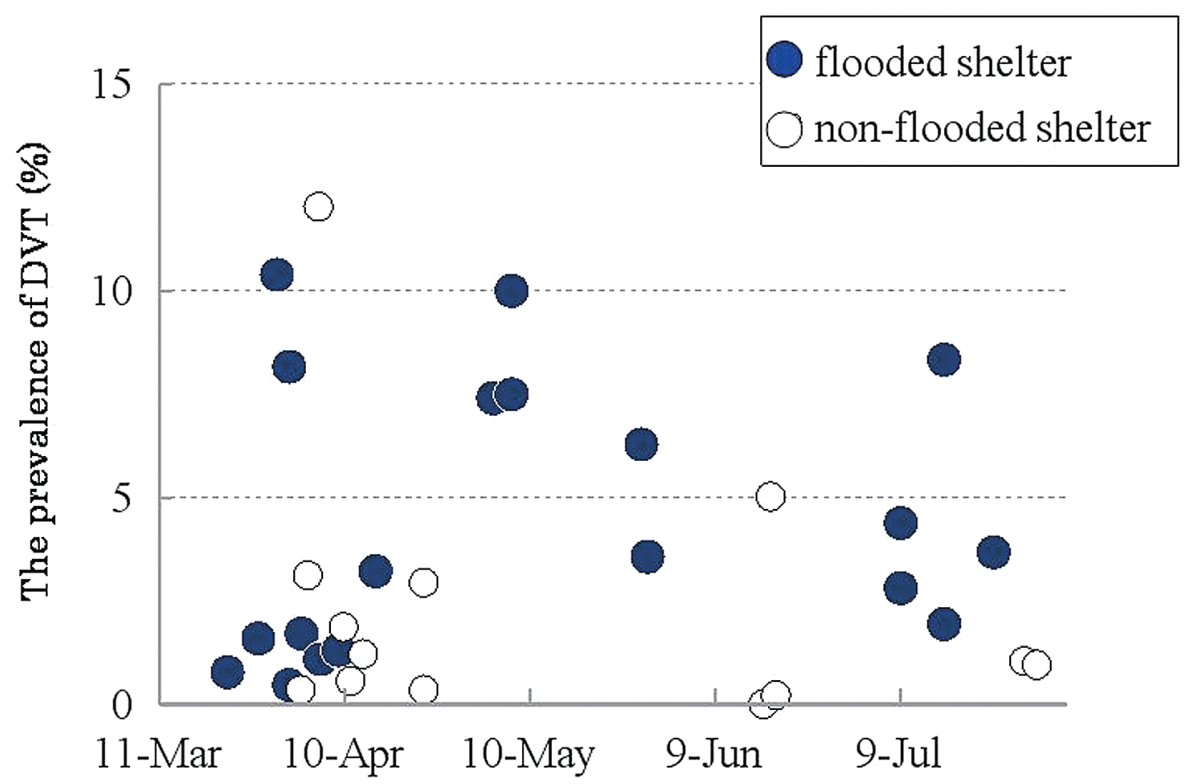

Fig. 2. Variability in the prevalence of deep vein thrombosis (DVT) in shelters over time after the earthquake. Dark circles represent the prevalence of DVT in tsunami-flooded shelters, while white circles represent that in nonflooded shelters. The total number of examinees was 701 out of 8,630 evacuees. The DVT prevalence in tsunamiflooded shelters seemed to decrease over time but remained higher than that in non-flooded shelters. The highest prevalence of DVT was observed on 6-April in a non-flooded shelter located on the hill, the shelter of which was extremely crowded with evacuees who fled from the fire of a flooded shelter in the evening of the earthquake on March 11.

Table 1. Prevalence of deep vein thrombosis (DVT) in flooded and nonflooded shelters.

$\left.\left.\begin{array}{lcc}\hline \multicolumn{1}{c}{\text { Prevalence of DVT }} & \text { March-May } & \text { June-July } \\ \hline \text { Flooded shelters } & 2.82 \% \\ & (109 / 3,871) \\ \text { Non-flooded shelters } & 1.68 \% \\ & (53 / 3,155)\end{array}\right\} P=0.0016 \quad \begin{array}{c}3.33 \% \\ (18 / 541) \\ 0.94 \% \\ (10 / 1,063)\end{array}\right\} P<0.001$

The prevalence of DVT (top) and actual number of patients per population of shelters (bottom) in the flooded and non-flooded shelters in the early phase (from March to May) and late phase (from June to July), respectively, are shown in each column. The prevalence of DVT in tsunami-flooded shelters is noticeably high in each phase. The statistical analysis was performed by using chi-square test.

for short-term stay (ie, a few days), and 40 square feet for long-term stay (ie, days to weeks). In the Great East Japan earthquake, most of the shelters, especially in the tsunamiflooded areas, provided 10 square feet per person for many weeks and at most 20 square feet per person for several months. Evacuees stayed in the crowded shelters for a long time, which would restrict their physical activity and facilitate stagnation of blood in their lower extremities. A similar mechanism that leads to DVT and PTE in occupants of shelters was described for casualties of the night air-attacks in London (Simpson 1940).

Our survey revealed a high prevalence of DVT in the shelters, especially in tsunami-flooded shelters, which has never been reported in the literature. In addition, evacuees in widely devastated and less accessible areas have multiple, serious risks for DVT. Therefore, we recommend not setting up long-term shelters in flooded areas after disasters.

\section{Conflict of Interest}

We declare that there are no conflicts of interest with this manuscript.

\section{References}

Atri, M., Herba, M.J., Reinhold, C., Leclerc, J., Ye, S., Illescas, F.F. \& Bret, P.M. (1996) Accuracy of Sonography in the evaluation of calf deep vein thrombosis in both postoperative surveillance and symptomatic patients. Am. J. Roentgenol., 166, 1361-1367.

FEMA (2008) Guidelines for design of structures for vertical evacuation from tsunamis, FEMA P646 Report, Federal Emergency Management Agency U.S. Department of homeland security, Washington D.C., pp. 57-58.

Inoue, K. (2006) Venous thromboembolism in earthquake victims. Disaster Manag. Response, 4, 25-27.

Philbrick, J.T. \& Becker, D.M. (1988) Calf deep venous thrombosis: a wolf in sheep's clothing? Arch. Intern. Med., 148, 2131-2138. 
Sakuma, M., Nakamura, M., Yamada, N., Ota, S., Shirato, K., Nakano, T., Ito, M. \& Kobayashi, T. (2009) Venous thromboembolism: deep vein thrombosis with pulmonary embolism, deep vein thrombosis alone, and pulmonary embolism alone. Circ. J., 73, 305-309.

Shibahara, S. (2011) The 2011 Tohoku Earthquake and Devastating Tsunami. Tohoku J. Exp. Med., 223, 305-307.
Simpson, K. (1940) Shelter death from pulmonary embolism. Lancet, 236, 744.

Watanabe, H., Kodama, M., Tanabe, N., Nakamura, Y., Nagai, T., Sato, M., Okabe, M. \& Aizawa, Y. (2008) Impact of earthquakes on risk for pulmonary embolism. Int. J. Cardiol., 129, 152-154. 